\title{
Apoio matricial como dispositivo do cuidado em saúde mental na atenção primária: olhares múltiplos e dispositivos para resolubilidade
}

\author{
Matrixing support as an instrument of primary healthcare \\ in mental health: multiple views and devices for resolution
}

\author{
Antonio Germane Alves Pinto ${ }^{1}$ \\ Maria Salete Bessa Jorge ${ }^{2}$ \\ Mardenia Gomes Ferreira Vasconcelos ${ }^{3}$ \\ José Jackson Coelho Sampaio ${ }^{2}$ \\ Gláucia Posso Lima ${ }^{4}$ \\ Valéria Carneiro Bastos ${ }^{4}$ \\ Helena Alves de Carvalho Sampaio ${ }^{4}$
}

\footnotetext{
${ }^{1}$ Universidade Regional do Cariri. Rua Cel. Antônio

Luiz 1161, Pimenta. 63105-000 Crato CE. germanepinto@hotmail.com ${ }^{2}$ Departamento de Saúde Pública, Centro de Ciências da Saúde, Universidade Estadual do Ceará

${ }^{3}$ Núcleo de pesquisa Saúde Mental, família, práticas de saúde e enfermagem,

Universidade Estadual do

Ceará

${ }^{4}$ Centro de Ciências da

Saúde, Universidade

Estadual do Ceará
}

\begin{abstract}
The scope of this paper is to analyze the articulation of the mental health services between the teams of the Family Health Strategy and Psychosocial Care Center by the matrixing process with emphasis on comprehensive care and case resolution. The subjects included 32 users and 22 family members attended in the matrixing in mental health, 46 health professionals from the Family Health Strategy and 15 from the Psychosocial Care Centers in two cities in the state of Ceara. It is a study of a qualitative nature based on critical hermeneutics. As dictated by the results, the integration of mental health services in Primary Healthcare establishes innovative approaches for shared psychosocial care between staff, family members and users. The matrixing activities in mental health ensure broadened access and diversification of healthcare to achieve comprehensive care. Case resolution is defined by the acknowledgement of the social conditions of demand by the teams and overcoming medicationbased health practices.
\end{abstract}

Key words Mental health, Primary Healthcare, Matrixing support, Unified Health System
Resumo Objetiva-se analisar a articulação das ações de saúde mental entre as equipes da Estratégia Saúde da Família e do Centro de Atenção Psicossocial pelo processo de matriciamento com ênfase na integralidade do cuidado e resolubilidade assistencial. Participaram 32 usuários e 22 familiares atendidos no matriciamento em saúde mental, 46 profissionais de saúde da Estratégia Saúde da Família e 15 dos Centros de Atenção Psicossocial de dois municípios do Estado do Ceará. Trata-se de um estudo de natureza qualitativa pautado na hermenêutica crítica. Conforme os resultados, a integração das ações de saúde mental na Atenção Primária em Saúde estabelece inovação das abordagens psicossociais pela assistência compartilhada entre equipe, familiares e usuários. As atividades do matriciamento em saúde mental disponibilizam ampliação de acesso e diversificação da atenção à saúde direcionada para a integralidade. A resolubilidade assistencial delimita-se pelo reconhecimento das condições sociais da demanda pelas equipes e na superação de práticas medicamentalizadas de saúde.

Palavras-chave Saúde mental, Atenção Primária à Saúde, Apoio matricial, Sistema Único de Saúde 


\section{Introdução}

Na consolidação do Sistema Único de Saúde (SUS), a Estratégia Saúde da Família (ESF) tornou-se um dispositivo estratégico para inversão do modelo assistencial curativo e hospitalocêntrico. Em suas diretrizes, focaliza a prevenção de doenças, o controle de agravos e a promoção da saúde. As ações devem ser operadas no contexto territorial e comunitário com atuação multidisciplinar e participativa ${ }^{1,2}$.

Concomitantemente, o Centro de Atenção Psicossocial (CAPS) apresenta-se como serviço de saúde ordenador das ações de saúde mental nas redes assistenciais. Atualmente, as necessidades e demandas da população requisitam uma articulação efetiva do cuidado em saúde mental no território. $\mathrm{O}$ processo de matriciamento em saúde mental ocorre na integração das equipes de saúde da família e atenção psicossocial para acompanhamento das pessoas com problemas psíquicos leves ${ }^{3-5}$.

O reconhecimento da dimensão subjetiva e social do usuário no desenvolvimento terapêutico no campo da saúde mental é decorrente de movimentos revolucionários no modo de olhar e de cuidar da pessoa com sofrimento psíquico e/ou transtorno mental. Dessa maneira, evidencia-se o sujeito e suas singularidades em contraposição ao agir voltado para a doença, por vezes, determinando práticas manicomiais e asilares ${ }^{6}$.

Evidentemente, a complexidade dos problemas de saúde mental exige a articulação entre as múltiplas formas assistenciais às quais o usuário recorre como subsídio para suas demandas e necessidades de vida. No campo da saúde mental, o território constitui o lugar onde as situações, as pessoas ou as relações mais complexas que envolvem o seu (des)equilíbrio estão presentes ${ }^{6,7}$.

Todavia, as tentativas de consolidação das redes assistenciais pelas políticas ou pelas iniciativas dos sujeitos participantes desse processo almejam a integralidade do cuidado na sua dimensão plena. Entretanto, as dificuldades mostram-se na própria efetivação das linhas de cuidado que estão fragmentadas em seus fluxos e conexões. Com isso, os resultados são itinerários terapêuticos pontuais, superficiais e desarticulados com as demandas e necessidades de saúde do usuário ${ }^{8-10}$.

Nesse sentido, objetiva-se analisar a articulação das ações de saúde mental entre as equipes da Estratégia Saúde da Família e do Centro de Atenção Psicossocial pelo processo de matriciamento com ênfase na integralidade do cuidado e na resolubilidade assistencial.

\section{Metodologia}

Trata-se de pesquisa com abordagem qualitativa numa perspectiva crítica e reflexiva ${ }^{11}$. Como cenários da pesquisa constaram dois municípios do Estado do Ceará, no Nordeste do Brasil. O cenário 1 é a capital do Estado, detentora de uma população de 2.505.552 habitantes e considerada a quarta capital do Brasil em densidade demográfica $^{12}$. No sistema municipal de saúde existem cinco redes assistenciais: básica, hospitalar, especialidades, saúde mental e urgência/emergência. Enquanto a cobertura da ESF é de 34\%, o número de CAPS é de quatorze, distribuídos nas seis áreas de saúde das Secretarias Executivas Regionais (SER) $)^{13,14}$.

Já o cenário 2 localiza-se no interior do Estado, na zona norte, e sua população é de 182.430 habitantes. O sistema municipal de saúde detém uma cobertura da ESF acima de $95 \%$ e os serviços de saúde mental compõem a Rede de Atenção Integral em Saúde Mental (RAISM) com CAPS Geral, CAPS AD, Residência Terapêutica e Leitos Psiquiátricos em Hospital Geral, bem como, estratégias de articulação comunitária.

Segundo mencionado, no cenário 1 os participantes foram 20 usuários, 10 familiares, 9 profissionais de saúde do Centro de Atenção Psicossocial e 22 da Estratégia Saúde da Família. No cenário 2, participaram 12 usuários, 12 familiares, 6 profissionais de saúde do Centro de Atenção Psicossocial e 24 da Estratégia Saúde da Família. Entre os profissionais, foram 19 médicos, sendo 7 psiquiatras, 21 enfermeiros, 10 psicólogos, 5 assistentes sociais, 4 terapeutas ocupacionais, 1 farmacêutico e 1 fisioterapeuta. No total, participaram 115 pessoas de ambos cenários.

A seleção dos grupos representativos do estudo ocorreu pela implicação deles na realidade investigada e a composição final do número de participantes respalda-se na saturação teórica sobre o objeto de estudo ${ }^{15}$.

Para a coleta dos dados, utilizaram-se as técnicas de grupo focal, a entrevista semiestruturada e a observação sistemática. Em cada técnica aplicada respaldaram-se o diálogo e a condução compartilhada para o reconhecimento da intersubjetividade e da implicação de cada participante.

De acordo com o determinado, cumpriramse os preceitos éticos da pesquisa envolvendo seres humanos ${ }^{16}$ conforme parecer do Comitê de Ética em Pesquisa da Universidade Estadual do Ceará (UECE).

Para a análise dos dados trabalhou-se com base nos pressupostos da hermenêutica crítica ${ }^{11}$. 
O processo de evidenciação das sínteses convergentes, divergentes e diferentes bem como suas complementaridades foi discutido e articulado com a literatura disponível na área de conhecimento.

\section{Resultados e discussão}

As práticas em saúde mental: o uso das tecnologias leves - acolhimento, vínculo, corresponsabilização e autonomia

Na rede de atenção à saúde do SUS, a produção do cuidado recorre à hegemônica prática assistencial fundamentada na operacionalização de procedimentos de cunho biologizante, quase sempre voltados para a cura ou a reabilitação. Dessa forma, o modelo de atenção baseado na promoção da saúde, preconizado na formulação das políticas públicas, contrasta com a realidade vivenciada no cotidiano das relações terapêuticas.

A consulta médica ainda é uma prática requisitada na gestão das demandas como única resolução. Para usuários e familiares, o atendimento médico significa um caminho para melhoria de sua condição de saúde. Consoante evidenciado, o cuidado operado no cotidiano da ESF se revela nas medidas prescritivas, procedimentos de controle e intervenções programáticas do processo saúde-doença, tais práticas incorporam ainda uma resistência para o atendimento de pessoas com problemas psíquicos.

Uma grande dificuldade que nós temosé na questão da participação dos profissionais [...] principalmente dos médicos. [Devido à demanda de atendimentos clínicos], para alguns coordenadores da unidade de saúde, tirar o médico da consulta é o fim do mundo! O mundo vai acabar! Vão morrer todos os pacientes! Então, nós temos uma dificuldade de fechar as agendas dos médicos no matriciamento, principalmente pela demanda da população que é muito grande e o acesso já é difícil. E a consulta psiquiátrica demanda um tempo bem maior e nem todo profissional tem essa disponibilização de tempo. (Profissional de saúde da ESF - Cenário 1)

A maior dificuldade ainda é uma certa resistência de alguns profissionais [da ESF] em atender a demanda de saúde mental. Talvez por conta já do dia-a-dia mesmo dos programas, que toma muito tempo, é programa de hipertensão, de diabetes, de gestante e do idoso. Leva muito tempo, mesmo (Profissional do CAPS - Cenário 1)

Eu gosto porque eles [médicos] passam medicamentos, mas a melhora é pouca. (Familiar Cenário 2)
$O$ atendimento é muito bom. Esse pessoal aí [equipe do matriciamento] já está com duas vezes que eu venho pra eles, na primeira vez, eles passaram um remédio e tenho ficado é bem. Tenho conseguido dormir. Eu vim dessa vez porque o meu medicamento acabou, mas agora eu já estou com a receita (Usuário - Cenário 1 )

Logicamente, os riscos e as vulnerabilidades da composição fisico-orgânica da vida humana em sociedade determinam o funcionamento dos serviços de saúde de modo relevante. Assim, as demandas de saúde da população prevalecem no déficit patológico do viver, ou seja, nos agravos, doenças e crises. Por conseguinte, as redes assistenciais são interpostas nesta premissa emergente de cuidar na dor e na enfermidade, reduzindo, por vezes, o espectro da prevenção e da promoção em saúde, mantendo a condição de psiquiatrizado do usuário ${ }^{17}$.

Concomitantemente, o usuário depara-se com entraves no fluxo de atenção nas redes assistenciais caracterizados como dificuldades para a integralidade do cuidado em saúde. Consoante divulgado, as filas e as listas de espera para atendimento individual e, muitas vezes, de avaliação inicial emperram a possibilidade de superação da concepção hierarquizada do conjunto de serviços de saúde do SUS. Fica difícil para as equipes concretizar uma abordagem mais integralizada, pois precisam dar conta de inúmeras demandas significativas para a saúde da comunidade ${ }^{5,18}$.

Em virtude de possuir um conjunto de atividades cotidianas pautadas na programação de áreas de atenção, a ESF constrói um espaço delimitado de atuação que prima pela atenção integral. No entanto, as particularidades encontradas em cada área técnica precisam interagir com intersecções no campo clínico comum a todo indivíduo, são ou doente ${ }^{3}$.

Especificamente, na abordagem dos casos de saúde mental o foco tende a ser o desequilíbrio psíquico demarcado pelos comportamentos não comuns para a vida. Os transtornos e seus níveis de gravidade entornam o atendimento segundo o qual tais sintomas assumem uma ordem de resolução ou encaminhamento. Sumariamente, as ações são voltadas para a manutenção do equilíbrio, se o caso for leve; ou, para o encaminhamento direto a serviços especializados, tais como o CAPS, para casos moderados ou graves.

Diante da articulação entre as ações de saúde mental e o processo assistencial da ESF, algumas transformações ocorrem neste formato de atendimento. O usuário se mantém privilegiando a consulta especializada por um lado, mas reco- 
nhecendo a importância da escuta, por outro. Já os profissionais reconhecem que as práticas são processuais por serem modificadas com as experiências vividas no matriciamento a cada dia.

Então o nosso usuário é muito ligado no especialista. [...] o usuário já vem se consultar pedindo pra ir para o psiquiatra. Daí a gente ter condições de fazer um vínculo com ele e começar o tratamento com um transtorno leve; é uma coisa que pra gente mesmo é meio desgastante, porque a gente vê a ânsia do usuário de querer falar só com o psiquiatra, por causa da nossa própria cultura, a cultura do médico especialista. (Profissional de saúde da ESF - Cenário 2)

[No matriciamento] o paciente tem a unidade próxima de casa e pode ser assistido como um todo. Pode ser assistido de uma forma geral, tanto da parte clínica como nessa parte [psicossocial] que antes era vista separada. [...] às vezes chega um hipertenso e a pressão dele está alterada. Não é nem o fator orgânico, não é porque o remédio não está funcionando, é porque às vezes ele está precisando ser ouvido, ele está com algum problema, é preciso ouvir e perguntar o que está acontecendo, como está sua vida, ou seja, dá uma abertura para ele começar a falar [...] estabelecendo a confiança [...] então a cada dia ele vai se abrindo mais, vai melhorar, vai depois expor certos problemas que antes ele não tinha coragem de falar. (Profissional de saúde da ESF - Cenário 1)

Até agora estamos sendo bem tratados e bem acompanhados. O médico é bem atencioso. Conversou, explicou e perguntou o que a gente achava que ele (usuário) tinha. Disse que era para ele ser acompanhado também pela terapeuta ocupacional, pela psicóloga, e até pela fonoaudióloga porque não consegue falar direito. E isso tá acontecendo. $E$ muito bom. (Familiar - Cenário 2)

Eu acho assim: esse apoio matricial é muito bom porque vêm muitos profissionais. Vem a psicóloga, o médico, tem uma lourinha que é eu num sei o que ela é [terapeuta ocupacional] e ainda tem também o pessoal daqui [Equipe da ESF]. (Familiar - Cenário 1)

É bom, né. O pessoal vem de fora. Atende a gente. É muita gente para ser atendido, mas eu fiquei esperando e depois pronto. Foi tudo muito tranqüilo, eles me deixaram bem a vontade e eu contei tudo, falei o que eu achava que era [problema de saúde mental]. (Usuário - Cenário 1)

É possível exercer uma clínica solidificada em saberes e práticas inovadoras com vistas a abordar de modo complexo os problemas emanados no sujeito-usuário em relação à sua subjetividade. Como proposto, o encontro entre a necessi- dade de se equilibrar psiquicamente, interagir socialmente com maior autonomia e efetivar projetos terapêuticos integrais pode ser possibilitado pela interposição de arranjos de trabalho e gestão que se transversalizem pela subjetividade e pela cultura ${ }^{4,19}$.

Inicialmente, as práticas voltadas para saúde mental na atenção primária em saúde são as atividades transversais. Assim, o enfoque dado aos problemas psíquicos deve permear as demais abordagens programadas para cada grupo populacional, situação de risco ou vulnerabilidade. $\mathrm{Na}$ rotina das equipes da ESF, este trabalho é realizado com dinâmicas em sala de espera, nas discussões coletivas e no diálogo individual em cada atendimento. No conteúdo de cada atendimento com ênfase nos aspectos da subjetividade e no equilíbrio mental do indivíduo estão as situações que o envolvem nas suas relações sociais e no enfrentamento do estresse cotidiano.

\section{Apoio matricial em saúde mental: arranjos e dispositivos no território da ESF}

Pauta-se no matriciamento em saúde mental um conjunto de concepções e modelos para a organização da atenção à saúde no SUS. Neste estudo, enfocam-se as unidades de análise discutidas pela literatura ${ }^{5,18-20}$ que facilitam o entendimento sobre este processo de consolidação das ações psicossociais na ESF, postas em destaque.

A demanda do SUS reflete as condições de vida da população e seus mecanismos de resolução. Por diversos determinantes, a relação oferta-demanda é tensa e conflituosa, pois a baixa cobertura dos serviços e a delimitada atuação regem uma fragmentação contínua do cuidado em saúde ${ }^{18,21,22}$.

Nos CAPS e na ESF, a triagem da demanda tenta equalizar o fluxo entre a chegada, a permanência e a saída do usuário dentro do serviço. Este processo, denominado de acolhimento, enfatiza uma escuta qualificada, mas com foco na situação clínica e na sua gravidade para resolução e/ou encaminhamento ${ }^{22}$.

Inegavelmente, muitas pessoas estão ausentes dos serviços, por diversos motivos que impedem sua entrada. Caracterizam a demanda reprimida pelo sistema. Para tanto, a atenção integral mobiliza a equipe para uma aproximação intersubjetiva, observando questões e condições da dimensão psicossocial, tanto nos aspectos socioeconômicos como também na condição clínica que facilitem o acesso e efetivem a resolução de queixas e necessidades. 
Agiliza. Sabendo fazer e sabendo se organizar. Se administrado corretamente, com certeza agiliza esse fluxo de atendimento. O matriciamento é algo que veio exatamente para ajudar as pessoas não ficarem somente com aquele estímulo de CAPS, porque hoje em dia todo mundo está sujeito a ter qualquer problema mental, ninguém é melhor do que ninguém. É preciso acabar os estímulos aos preconceitos, então, a partir do momento que a gente estiver atendendo o paciente do CAPS no matriciamento permanente nos postos de saúde, vai acabar com isto. (Profissional de saúde do CAPS - Cenário 1)

Melhora os acompanhamentos, melhora, as sim, até o nosso entendimento com relação à doença, quando outros profissionais estão envolvidos a gente acaba, como enfermeiro, a gente aprende a lidar melhor com aquele paciente, a entender a doença dele de uma maneira mais satisfatória e pode dar uma qualidade de vida melhor para o paciente, ajudar no tratamento dele de várias formas. Com certeza melhorou a visão do profissional que pode intervir na área dele para ajudar num benefício, numa coisa que ajuda a família, com o acompanhamento do psicólogo, ajudando a família, no tratamento do paciente, na melhoria do paciente, e acho isso superimportante, quando não tinha [o matriciamento], o paciente ficava só no tratamento medicamentoso. (Profissional de saúde da ESF - Cenário 2)

É bom também porque é perto, eu moro bem aqui [Perto da Unidade da ESF]. Uma vez eu fui atrás lá [no CAPS], mas não dá certo, não. É muito longe. E a fila para ser atendido é grande. (Usuário - Cenário 1)

Mudou para melhor, as consultas dele [usuário] era no CAPS. Era dispendioso porque nem toda consulta, nós tínhamos dinheiro para pagar moto-taxi que era $R \$ 8,00$. Aí, eu conversei lá [CAPS] perguntando se podia ficar aqui no posto e eles disseram que podia. Só se o caso for muito grave, é que levo ele para o CAPS. E assim tá dando certo. (Familiar - Cenário 2)

Em contraste com a formação recebida pelas equipes de saúde, conforme revelam os depoimentos, as necessidades sociais dos usuários somente são dispostas nas condutas clínicas em saúde quando observadas pela dialogicidade do encontro entre eles. Nesse sentido, é necessário, então, planejar o projeto terapêutico singular e integral que conforma as intenções, estratégias e intervenções da equipe para com os agravos e problemas e, principalmente, a participação do usuário, agora sujeito, na resolução das suas demandas e necessidades ${ }^{18-20,22}$. Tal evidência recorre nas falas dos participantes:
Ainda falta as pessoas se conhecerem mais. Conhecer um pouco como é que funcionam os dois serviços. Saber o que é que a rede dispõe. O que a rede tem disponivel para oferecer para o usuário. Saber o que encaminhar. Porque às vezes os profissionais não sabem nem como é que funciona o serviço. (Profissional de saúde do CAPS - Cenário 1)

[...] de uma maneira geral, tanto profissionais de saúde, seja médico, enfermeiro, auxiliar de enfermagem, técnico de enfermagem, agentes de saúde, como profissionais da limpeza da unidade de saúde devem aprender a respeitar os pacientes que têm transtornos mentais. Aprendam a ter uma melhor abordagem, conversar melhor recepcionálos melhor, entendeu? E olhar, realmente a relação ética que tem que ter com o paciente. (Profissional de saúde da ESF - Cenário 2)

É tem sido bom sim. Mas eu queria era mesmo era ficar boa. Tem muita conversa, fico é nervosa, pois sempre tenho que falar toda vez, até parece que eles não sabem, não tem anotado lá. Eu fico nervosa porque eu tava esperando, é muita gente né?! Mas eu acho que eles se comprometem sim. Eles combinam um com os outros o que é que vão fazer. Eu gosto muito, uns às vezes faz pergunta e depois também fazem uma sugestão. Já me encaminharam para a terapia, o negócio é que eu nunca fui. (Usuário - Cenário 2)

Olha, eu já falei até pra ele [médico] mesmo. Eu já parabenizei ele duas vezes. É um profissional muito bacana. Acolhe a gente! Porque, mas tem médico que num se empenha tanto, num recebe direito. Mas tem aqueles que ajudam a gente resolver, né! Principalmente nesses casos [de saúde mental]. (Familiar - Cenário 1)

$\mathrm{Na}$ efetividade do seu processo de cuidado, o dispositivo da corresponsabilização entre equipe e usuário incorpora a família, a comunidade e as ações intersetoriais articuladas no contexto sociocomunitário. O diferencial deste dispositivo terapêutico se pauta no compartilhamento de problemas vividos e na pactuação integrada das resoluções. À medida que todos assumem a responsabilidade para a melhoria das condições de vida, seja individual ou coletiva, os resultados são alcançados de modo mais resolutivo. Os familiares e usuários dispõem de iniciativas mútuas juntamente com a equipe que favorecem a assistência integral e o acesso ao serviço de saúde. No entanto, o envolvimento compartilhado exige a efetiva participação do usuário em seu processo de restabelecimento do equilíbrio.

Geralmente, os pacientes vêm acompanhados da família, sempre tem uma responsável pela medicação. O paciente que vem sozinho são aqueles 
com problemas menores, que não tem gravidade; mas o paciente mais grave que não tem noção da medicação, do controle do tratamento, ele vem sempre acompanhado, e não é entregue a medicação para ele sozinho, sempre a gente pede ao responsável pelo paciente, tem sido sempre assim. Agora, as pessoas que têm consciência, as pessoas que estão bem no tratamento, elas vêm sozinhas. (Profissional de saúde da ESF - Cenário 2)

Mas a gente vê que para alguns pacientes a mudança é realmente grande e muito valiosa para eles. [É assim] de você não precisar se deslocar, de você se deslocar no meio em que você vive, de te abrir as portas da unidade básica de saúde e de saber que lá você vai ser recebido. Ao contrário de antigamente que era aquela coisa de colocar na ambulância e de mandar ou pro CAPS ou para o hospital, lá é um espaço onde você vai ser acolhido, também. Para boa parte dos pacientes essa mudança é grande, em todos os atendimentos. (Profissional de saúde do CAPS - Cenário 2)

Às vezes o meu filho fica agressivo e com os medicamentos ele melhora. Os médicos aqui se preocupam muito com a gente. Meu filho não queria tomar mais remédio, a depressão dele voltou muito forte, ele andava conversando só e eu vim pra cá. No mesmo dia que vim atenderam logo e aí graças a Deus ele está bem. Marcaram para eu trazer ele novamente hoje e estou aqui. Quando preciso, eles me atendem. E eu sempre acompanho meu filho. sigo as orientações daqui. (Familiar - Cenário 2)

Eu reconheço que não depende da vontade só dos profissionais, é mais minha do que deles.(Usuário - Cenário 1)

Pelo visto, no cotidiano da ESF é relevante o número de situações que revelam o uso inadequado de medicação. Ainda hoje, o processo de medicalização e medicamentalização na sociedade detém significativo valor na resolução de problemas pessoais entre os usuários ${ }^{23}$. Em casos de sintomas psíquicos, o equilíbrio mental é, por vezes, buscado pelo uso contínuo de medicamentos que requisitam uma administração controlada e/ou supervisionada.

Em situações de crise psíquica, as intervenções ainda sugeridas para o usuário remetem a uma composição institucional parcializada, ou seja, as abordagens iniciais são trocadas por resoluções imediatas de transferência e encaminhamento para unidades especializadas, principalmente para instituições hospitalares de emergência em psiquiatria. $\mathrm{Na}$ atenção primária em saúde, a iniciativa de acolhimento e o vínculo estabelecido na condução terapêutica já favorecem a execução de um fluxo contínuo na assistência requisitada.

\section{Resolubilidade do apoio matricial em saúde mental: da demanda às necessidades de saúde}

Como observado, a dispensação de medicamentos psicotrópicos e o consequente controle requerido para tal atividade constituem o principal registro da atuação na área psiquiátrica da ESF. Acrescentam-se os registros de referência e contrarreferência para unidades especializadas e hospitalares. Sendo assim, a demanda atendida na ESF revela pouco da situação real vivenciada pela população no tocante ao sofrimento psíquico.

Portanto, é no território onde a população efetiva seu cuidado e, por isso, os dispositivos assistenciais do SUS devem se articular com tais instituições para compor pactos de atuação integrada. Com o apoio matricial, a integração incide na interlocução entre CAPS e ESF, sobretudo. Dessa maneira, o trabalho das equipes do matriciamento compartilham informações territoriais, demandas clínicas e de procedimentos bem como ampliam seu potencial resolutivo perante os casos clínicos de saúde mental.

A gente [equipe] tem um sistema nessa unidade [ESF] de renovação de receitas controladas, então, toda vida que o paciente busca essa renovação é feita uma revisão do prontuário dele pra gente ver se ele está em acompanhamento médico ou não. Quando a gente percebe que há muito tempo ele não é visto, a gente faz a "busca ativa", o paciente vem pra cá, a gente colhe uma nova história, reavalia e, muitas vezes, nessa situação, a gente acaba captando pacientes da área que são necessários, os que têm a necessidade de serem vistos no matriciamento. (Profissional de saúde do CAPS - Cenário 2)

Visualizando o que a gente sempre faz aqui, que é a questão da territorialização, conhecer os equipamentos existentes é o passo que é catalogar tudo o que existe porque pra você desenvolver alguma coisa você tem que saber o que existe na sua comunidade [...] É um grupo da própria associação do bairro, é um grupo de idoso, de caminhada, do skate, de lazer na comunidade, então é ver o que tem e tentar ampliar fazendo parcerias. A assistência tem começado a se construir nos territórios. (Profissional de saúde do CAPS - Cenário 2)

Então, assim, com certeza depois do matriciamento eu tô mais sensibilizada, já dou mais atenção, escuto melhor, nem tudo precisa estar indo pro CAPS, realmente não, muitas vezes o paciente quer apenas ser ouvido, acolhido, e isso dá pra gente fazer aqui. (Profissional de saúde da ESF - Cenário 1)

Assim, eu acho que melhorou bastante. Eu acho que eles [equipe] fizeram uma reciclagem, porque 
antes eu vinha e num era bem atendida não. Nem escutavam a gente direito. Ficava muito a desejar. Melhorou, agora eu venho, converso e me sinto bem melhor. (Usuário - Cenário 1)

Acho que não está me ajudando porque meu filho continua quase do mesmo jeito, a melhora foi muito pouca, estou até sem trabalhar para ficar com ele porque não tem ninguém que queira ficar com ele. Eu não sei o que vou fazer porque não tenho marido, tenho que trabalhar para pagar minhas contas, não tenho casa, moro de aluguel, pago água, luz e as coisas do meu filho. Até agora acho que as minhas necessidades não estão sendo resolvidas aqui porque se tivesse ajudado ele estaria um pouco mais quieto e estou achando que ele está é mais inquieto. Mas só que antigamente ele não dormia bem, era agitado, agora ele está dormindo melhor. (Familiar - Cenário 2)

$\mathrm{Na}$ execução cotidiana, ocorrem grupos operativos e/ou ações de educação em saúde onde a congruência interdisciplinar é revelada pelas atitudes comuns de cada Profissional de saúde e percebida por usuários e familiares como algo positivo para sua melhoria de vida.

Em processo inovador, as abordagens clínicas se horizontalizam no modo de subjetivação do usuário, reconhecendo seu lugar existencial, subjetivo e, principalmente, social. A multiplicidade proporcionada pela inclusão multidisciplinar das equipes do CAPS e ESF remete a ações cada vez mais complexas e diversificadas.

Neste prisma, a ampliação dos serviços pelo aumento da cobertura assistencial bem como a disponibilidade de atividades terapêuticas apenas possibilitou o atendimento inicial do estreito ápice das demandas em saúde mental. O matri- ciamento tem como objetivo fortalecer o cuidado em saúde mental no território ${ }^{24}$, mas para alcançar sua meta é preciso resoluções equânimes e resolutivas que exigem também políticas sociais de inclusão e convivência social saudável na direção das necessidades de saúde do usuário, estas últimas mais complexas e abrangentes.

\section{Considerações finais}

$\mathrm{Na}$ integração do campo psicossocial no processo operacional da ESF, inegavelmente o cuidado em saúde mental ocorre na assistência direta das equipes de saúde com a participação dos usuários e familiares durante o processo terapêutico. A unidade de saúde configura um ponto de partida, passagem ou saída para o usuário na sua trilha para a resolução de problemas subjetivos e sociais. No entanto, as condições sociais, econômicas e culturais e o atrelamento a práticas medicamentalizadas reduzem a interlocução intersetorial e comunitária essencial para a atenção psicossocial.

Portanto, o apoio matricial em saúde mental opera práticas inovadoras e focos de atuação multidisciplinar. Nos fluxos assistenciais, a melhoria da articulação entre os serviços foi dinamizada entre o CAPS e as unidades de Saúde da Família. Constituir uma atenção integral à saúde, reconhecendo o campo psicossocial como enfoque indispensável em todas as ações assistenciais e de promoção exige uma composição gerencial, clínica, ética e política que priorize a intersubjetividade, a participação e a articulação intersetorial.

\section{Colaboradores}

AGA Pinto, MSB Jorge e MGF Vasconcelos trabalharam na pesquisa, metodologia e redação final. JJC Sampaio, GP Lima, VC Bastos e Sampaio HAC trabalharam na redação final. 


\section{Referências}

1. Brasil. Ministério da Saúde (MS). Secretaria de Assistência à Saúde. Coordenação de Saúde da Comunidade (MS). Saúde da Família: uma estratégia para a reorientação do modelo assistencial. Brasília. Ministério da Saúde (MS); 1997.

2. Brasil. Ministério da Saúde (MS). Departamento de Atenção Básica. Atenção Básica e Saúde da Família [site na Internet]. 2011 [acessado 2011 set 22]. Disponível em: http://dab.saude.gov.br/atencaobasica.php

3. Caçapava JR, Colvero LA. Estratégias de atendimento em saúde mental nas unidades básicas de saúde. Rev. Gaúch. Enferm 2008; 29(4):573-580.

4. Figueiredo MD, Campos RO. Saúde mental na atenção básica à saúde de Campinas, SP: uma rede ou um emaranhado? Cien Saude Colet 2009; 14(1):129138.

5. Nunes M, Juca VJ, Valentim CPB. Ações de saúde mental no Programa Saúde da Família: confluências e dissonâncias das práticas com os princípios das reformas psiquiátrica e sanitária. Cad Saude Publica 2007; 23(10):2375-2384.

6. Amarante P. Saúde Mental e Atenção Psicossocial. Rio de Janeiro: Fiocruz; 2007.

7. Alves DSN, Guljor AP. O cuidado em saúde mental. In: Pinheiro R, Mattos RA, organizadores. Cuidado: as fronteiras da integralidade. São Paulo, Rio de Janeiro: Hucitec, IMS-UERJ, Abrasco; 2003. p. 221-240.

8. Matumoto S, Fortuna CM, Mishima SM, Pereira MJB, Domingos NAM. Supervisão de equipes no Programa de Saúde da Família: reflexões acerca do desafio da produção de cuidados. Interface Comun Saúde Educ 2005; 9(16):9-24.

9. Franco TB, Magalhães Júnior HM. Integralidade na assistência à saúde: a organização das linhas do cuidado. In: Merhy EE, Magalhães Júnior HM, Rimoli J, Franco TB, Bueno WS, organizadores. O trabalho em saúde: olhando e experienciando o SUS no cotidiano. 2a ed. São Paulo: Hucitec; 2004. p. 125-134.

10. Machado MFAS, Monteiro EMLM, Queiroz DT, Vieira NFC, Barroso MGT. Integralidade, formação de saúde, educação em saúde e as propostas do SUS: uma revisão conceitual. Cien Saude Colet 2007; 12(2):335-342.

11. Minayo MCS. O desafio do conhecimento: pesquisa qualitativa em saúde. $11^{\text {a }}$ ed. São Paulo: Hucitec; 2008.

12. Brasil. Prefeitura Municipal de Fortaleza. Informações sobre a cidade. [site na Internet]. 2011 [acessado 2011 maio 31]. Disponível em: http://www.fortaleza. ce.gov.br

13. Brasil. Prefeitura Municipal de Fortaleza. Secretaria Municipal de Saúde. Relatório de Gestão 2007: Saúde, qualidade de vida e a ética do cuidado. Fortaleza: Imprensa Municipal; 2009.
14. Brasil. Ministério da Saúde (MS). Departamento de Atenção Básica. Cobertura da Estratégia Saúde da Família: credenciamento, implantação e funcionamento [site na Internet]. 2011 [acessado 2011 maio 31]. Disponível em: http://dab.saude.gov.br/historico_ cobertura_sf.php

15. Flick U. Introdução à pesquisa qualitativa. $3^{\mathrm{a}}$ ed. Porto Alegre: Artmed-Bookman; 2009.

16. Brasil. Ministério da Saúde (MS). Conselho Nacional de Saúde. Resolução no 196/96 sobre pesquisa envolvendo humanos. Brasília: Editora MS; 2000.

17. Pinto ATM, Ferreira AAL. Problematizando a reforma psiquiátrica brasileira: a genealogia da reabilitação psicossocial. Psicol. Estud. 2010; 15(1):27-34.

18. Arce VAR, Sousa MF, Lima MG. A práxis da Saúde Mental no âmbito da Estratégia Saúde da Família: contribuições para a construção de um cuidado integrado. Physis 2011; 21(2):541-560.

19. Camuri D, Dimenstein M. Processos de trabalho em saúde: práticas de cuidado em saúde mental na Estratégia Saúde da Família. Saude Soc. 2010; 19(4): 803-813.

20. Onocko Campos R, Gama CA. Saúde mental na Atenção básica. In: Campos GWS, Guerrero AVP, organizadores. Manual de Práticas em Atenção Básica: saúde ampliada e compartilhada. São Paulo: Hucitec; 2008. p. 209-235.

21. Pinto AGA, Jorge MSB. Prática clínica em saúde mental no cotidiano do Centro de Atenção Psicossocial. Cogitare Enfermagem 2009; 14(2):217-226.

22. Jorge MSB, Pinto DM, Quinderé PHD, Pinto AGA, Sousa FSP, Cavalcante CM. Promoção da Saúde Mental - Tecnologias do Cuidado: vínculo, acolhimento, co-responsabilização e autonomia. Cien Saude Colet 2011; 16(7):3051-3060.

23. Tesser CD. Medicalização social (II): limites biomédicos e propostas para a clínica na atenção básica. Interface Comun Saúde Educ 2006; 10(20):347-362.

24. Mielke FB, Olchowsky A. Saúde mental na Estratégia Saúde da Família: a avaliação de apoio matricial. Rev. Bras. Enferm. 2010; 63(6):900-907.

Apresentado em 28/09/2011

Aprovado em 29/10/2011

Versão final apresentada em 18/11/2011 